\title{
A STUDY ON MOVIE RECOMMENDER SYSTEMS BASED ON WORDPRESS PLATFORM
}

\author{
Tran Thi Thanh ${ }^{*}$ 四 \\ ${ }^{*}$ Faculty of Electronic Engineering, Thai Nguyen University of Technology, Thai Nguyen \\ University, Thai Nguyen 250000, Vietnam
}

DOI: https://doi.org/10.29121/ijetmr.v7.i6.2020.709

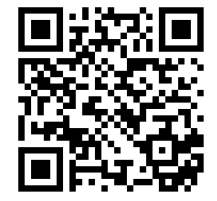

Article Citation: Tran Thi Thanh. (2020). A STUDY ON MOVIE RECOMMENDER SYSTEMS BASED ON WORDPRESS PLATFORM. International Journal of Engineering Technologies and Management Research, 7(6), 152-155. https://doi.org/10.29121/ijetmr.v7 i6.2020.709

Published Date: 28 June 2020

Keywords:

Recommender Systems

Movie Recommender Systems Information Filtering Data

\section{ABSTRACT}

The rapid growth of data collection has led to a new era of information. Data is being used to create more efficient systems and this is where Recommendation Systems come into play. Recommender systems are among the most effcient tools for information filtering to improve the quality of search results and provide items that are more relevant to the search item or are realted to the search history of the user, especially from big data on Internet. Among those, movie recommendation systems are the useful tools to assist users in classifying them with similar interests. This makes them a central part of websites and e-commerce applications. This paper aims to describe the implementation of a movie recommender system built on the Wordpress platform to be able to take advantage of the plugin support system and outstanding management and statistical features. The obtained results indicate that the proposed approach may provide high performance regarding reliability, efficiency, and accuracy. Moreover, the user-friendly interface and suitable display for devices ranging from desktop to mobile devices are also the advantages.

\section{INTRODUCTION}

The internet is one of the most important inventions of the modern era. As internet connectivity becomes ever more important, new technologies are emerging to connect the entire globe with better, faster service. The rapid development of internet has promoted the explosive growth of available information over the last decade. Hence, the demand for information filtering is more and more necessary. Recommendation systems (RS) is one of the most successful information filtering applications and has become a high efficient method to solve the information overload problem [1]. RS aims to automatically generate suggested items such as movies, books, journals, news, music, webpages, etc. for users based on their historical search. There are many studies on recommendation systems using different methods. A hybrid model-based movie recommendation system using the improve $\mathrm{K}\urcorner$-means clustering coupled with genetic algorithms (GAs) to partition transformed user space is done in [1]. The authors utilized pricipal component analysis (PCA) data reduction technique to densethe movie population space in order to reduce the computation complexity in intelligent movie recommendation. The obtained results showed that, compared with the previous studies, high performance in accuracy is achieved and more reliable and personalized movie recommendations are generated. In another study on an effective collaborative movie recommender system with cuckoo search [2], the authors concentrated on the movie recommendation system whose primary object aims to suggest a recommender system through data clustering and computational intelligence. The use of k-means clustering by adopting cuckoo search optimization algorithm is made by using the novel recommender system. The

(C) 2020 The Author(s). This is an open access article distributed under the terms of the Creative Commons Attribution License, which permits unrestricted use, distribution, and reproduction in any medium, provided the original author and source are credited. 
experimental results indicated that high performance in terms of reliability and efficiency. The dynamical modeling and simulation analysis of the recommendation on the user-movie network was done to characterize the personal search and the system recommendation mechanism [3]. The authors concluded that the change of the movie degree is different, and is related to the initial degree of movies, the adjacency matrix. Moreover, the advantage of the dynamical method is good in short or long time. A hybrid recommender system for recommending relevant movies using an expert system was proposed in [4]. The results of this work are that the system verification based on standard metrics (precision, recall, F1-measure) achieves results over 80\%. A complex hybrid system in the area of movie recommendation is created and verified on a group of users using the MovieLens dataset and compared with other traditional recommender systems. In another study, the authors presents Moreopt which is a web-based movie recommender system to overcome the sparsity and increase the density while remaining the same maximizing accuracy [5]. On the other hand, recommendation systems are also used in many other fields and exhibit many applications [6], [7], [8]. However, the recommendation systems in many movie websites do not work so well as we expected. Despite the large number of users, the common point of these sites is that they have not focused on movie recommendations for users. Typically when users view the information of a certain movie, the site will suggest movies with similar titles to the selected film, leading to the suggestions for users having no individuality and the accuracy is also not high.

In some other websites, the suggestion system only shows movies with large viewership for the week or new cinemas updated in the database. The results of this suggestion are more advanced because at least it is based on the level of interest of users to a movie expressed by the number of views, however, not all users like the "hot" movie or new movies coming out in theaters. In order to improve the accuracy and increase the personalization of the suggested results, this paper aims to study and develop a recommendation system for movie websites.

\section{MATERIALS AND METHODS}

The movie website will be built on the Wordpress platform to be able to take advantage of the plugin support system and outstanding management and statistical features.

For the movie website model, the users are divided into two categories: (1) the user, called members, has an account registration distinguished by user_id and has relevant information stored in the database; (2) the users, called visitor, do not register an account and just watch the movie introduction. After selecting any movie to read the introduction, only registered members can comment. The rating ranges from 1 to 5 stars, with 1 star being the least favorite and 5 stars being the most favorite.

\section{RESULTS AND DISCUSSIONS}

In the database, the following tables will be considered: 1).

wp_comments: store the comments for each movie and the id of the users who commented on that movie (Table

Table 1: wp_comments to store the comments for each movie and the id of the users

\begin{tabular}{|c|c|c|}
\hline Comment_ID & Comment_post_ID & User_ID \\
\hline 1 & 1 & 0 \\
\hline 2 & 16 & 1 \\
\hline 3 & 70 & 1 \\
\hline 4 & 29 & 1 \\
\hline 5 & 22 & 2 \\
\hline
\end{tabular}

wp_commentmeta: store favorite values, which mean the number of stars that each user has rated in each comment (Table 2).

Table 2: $w p_{-}$commentmeta to store favorite values

\begin{tabular}{|c|c|c|c|}
\hline Meta_id & Comment_id & Meta_key & Meta_value \\
\hline 1 & 2 & rating & 5 \\
\hline 3 & 3 & rating & 3 \\
\hline
\end{tabular}


Tran Thi Thanh

\begin{tabular}{|l|l|l|l|}
\hline 5 & 4 & rating & 5 \\
\hline 7 & 5 & rating & 5 \\
\hline
\end{tabular}

From the two tables above, view 'wp_user_rating' is created to query the necessary information (Table 3) including:

- comment_post_ID: ID of the film has been commented.

- user_id: ID of the user who commented on the movie.

- meta_value: how many stars the users have rated movies. In Wordpress, there are two meta_value values: rating and verified. Here the author only cares about rating values.

- (meta_key = "rating").

Table 3: Comment_post_ID, User_id, meta_value for building Utility matrix

\begin{tabular}{|c|c|c|}
\hline Comment_post_id & User_id & Meta_value \\
\hline 16 & 1 & 5 \\
\hline 70 & 1 & 3 \\
\hline 29 & 1 & 5 \\
\hline 22 & 2 & 5 \\
\hline 26 & 2 & 5 \\
\hline
\end{tabular}

For a new comment from the member, the data will be automatically updated into the view 'wp_user_rating'. Based on this view, the Utility matrix will be built to calculate similarity among the reviewed movies. The utility matrix is stored in the 'wp_predict' table with columns:

- item_id1: id of the movie reviewed.

- $\quad$ item_id2: id of the movie calculated with similarity to the movie with id of item_id1.

- times: Number of users rated both item_id1 and item_id2

- rating: Average deviation of rating value of item_id1 and item_id2.

After obtaining the Utility matrix, the Slope One algorithm is used to complete the missing values, thereby using the SQL query to find ids of the movies that can be suggested to the current user. 4):

Here is an example of the Utility matrix for item_id = "16" after the calculation from view `wp_user_rating` (Table

Table 4: An example of the Utility matrix for item_id = "16"

\begin{tabular}{|c|c|c|c|}
\hline Item_id1 & Item_id2 & Times & Rating \\
\hline 16 & 22 & 1 & 0 \\
\hline 16 & 29 & 1 & 0 \\
\hline 16 & 70 & 1 & 2 \\
\hline 16 & 94 & 1 & 2 \\
\hline
\end{tabular}

The recommendation system based on ratings has the disadvantage that it only applies to users who have reviewed movies or movies that have been pre-rated, so what do we have to suggest to visitors? There are two options:

- If a visitor sees the introductory information of a movie without any comments, the system will recommend visitors to the movies of the same genre. For example, you have just seen the introduction of the film Black Panther in the genre of action-fiction film, the system will suggest you the movie Gotham, Three billboards, and so on, which are also the kind of action films.

- If visitors view information of a movie that has already been commented, the system will recommend them the movies that have the highest similarity to that movie. 


\section{CONCLUSIONS}

In this article, a movie recommender system is built on the Wordpress platform. The performance of this approach is measured by regarding the accuracy, reliability and personalized movie recommendation systems. Also this method has user friendly interface and suitable presentation for devices ranging from desktops to mobiles. Movie data is updated regularly and fully. Suggestion algorithm brings out highly accurate suggestions to each different user. However, the Utility Matrix must be recalculated with every new evaluation data updated. It increases the recommended loading time for each movie. When the input data is large, Slope One algorithm will take time to build Utility matrix. Item-based Collaborative Filtering suggestion algorithm often has a "cold-start" problem. The system cannot find suggestions for movies that have never been rated by a user.

In future work, user clustering algorithm will be applied to increase the accuracy of Slope One algorithm when the amount of data is large. Applying suggestion algorithms is based on latent feedback to increase the accuracy of the system. the algorithm is needed to optimize in order to reduce the utility matrix calculation time when the amount of input data increases.

\section{SOURCES OF FUNDING}

None.

\section{CONFLICT OF INTEREST}

None.

\section{ACKNOWLEDGMENT}

The work presented in this paper is supported by Thai Nguyen University of Technology, Thai Nguyen University, Vietnam.

\section{REFERENCES}

[1] Wang, Z., Yu, X., Feng, N., \& Wang, Z.. An improved collaborative movie recommendation system using computational intelligence. Journal of Visual Languages \& Computing, 25(6), 2014, 667-675.

[2] Katarya, R., \& Verma, O. P.. An effective collaborative movie recommender system with cuckoo search. Egyptian Informatics Journal, 18(2), 2017, 105-112.

[3] Zhang, S., Jin, Z., \& Zhang, J.. The dynamical modeling and simulation analysis of the recommendation on the user-movie network. Physica A: Statistical Mechanics and Its Applications, 463, 2016, 310-319.

[4] Walek, B., \& Fojtik, V.. A hybrid recommender system for recommending relevant movies using an expert system. Expert Systems with Applications, 2020, 113452.

[5] Inan, E., Tekbacak, F., \& Ozturk, C.. Moreopt: A goal programming-based movie recommender system. Journal of Computational Science, 28, 2018, 43-50.

[6] Herce-Zelaya, J., Porcel, C., Bernabé-Moreno, J., Tejeda-Lorente, A., \& Herrera-Viedma, E.. New technique to alleviate the cold start problem in recommender systems using information from social media and random decision forests. Information Sciences, 2020. doi: 10.1016/j.ins.2020.05.071

[7] Ojagh, S., Malek, M. R., Saeedi, S., \& Liang, S.. A location-based orientation-aware recommender system using IoT smart devices and Social Networks. Future Generation Computer Systems, 108, 2020, 97-118.

[8] Quijano-Sánchez, L., Cantador, I., Cortés-Cediel, M. E., \& Gil, O.. Recommender systems for smart cities. Information Systems, 2020, 101545. doi:10.1016/j.is.2020.101545 